\title{
Tropical Fruit Wastes as an Organic Nutrient Sources for the Cultivation of Chlorella vulgaris and Haematococcus pluvialis
}

\author{
Y.H. Tan*, Y.J. Khoo**, M.K. Chai*† and L.S. Wong** \\ *College of Engineering, Universiti Tenaga Nasional, Jalan Ikram-Uniten, Kajang, Selangor 43000, Malaysia \\ **Faculty of Health and Life Science, INTI International University, Persiaran Perdana BBN, Putra Nilai, Nilai, Negeri \\ Sembilan 71800, Malaysia \\ †Corresponding author: M.K. Chai; mkchai@uniten.edu.my
}

Nat. Env. \& Poll. Tech.

Website: www.neptjournal.com

Received: 30-03-2020

Revised: 02-05-2020

Accepted: 25-06-2020

Key Words:

Chlorella vulgaris

Haematococcus pluvialis

Fruit waste

Organic nutrient source

\begin{abstract}
The possibility of replacing the inorganic medium with tropical organic fruit waste medium as a nutrient supplement was evaluated for the cultivation of Chlorella vulgaris and Haematococcus pluvialis in this study. Various concentrations of tropical fruit waste medium such as papaya, pineapple and mango were prepared to cultivate microalgae of $C$. vulgaris and $H$. pluvialis. The biomass concentration, productivity and specific growth rate were determined and compared with those grown in a fully inorganic medium. For C. vulgaris, the use of a $20 \%$ tropical fruit waste medium was found to yield higher biomass concentration (4.133-4.533 g/L) compared with cultivation in a fully inorganic medium (3.400 g/L). For $H$. pluvialis, the use of a $10 \%$ mango waste medium was found to yield a similar biomass concentration compared with cultivation in a fully inorganic medium $(3.400 \mathrm{~g} / \mathrm{L})$. These results unveiled the potential of utilizing with tropical organic fruit waste medium as an effective strategy to reduce the cultivation cost of microalgae and treat the tropical fruit waste prior to discharge to the environment.
\end{abstract}

\section{INTRODUCTION}

The economic growth and population growth have rendered fast-growing global energy demand. Currently, energy generation through fossil fuels combustion is still incumbent in most developing countries (International Energy Agency 2019) albeit they have creaked about many environmental problems such as global warming stemming from greenhouse gases emission (BP 2019). Besides, overreliance on nonrenewable fossil fuel accelerates resource depletion and increases economic burden due to the fluctuating price of fossil fuel. Given this scenario, alternative energy sources which are sustainable and greener have been proposed. Wellknown biofuel such as biodiesel has been recognized as a potential energy source as compared to fossil fuels (Leong et al. 2018).

Biofuels can be derived from renewable sources including plant or microalgae (Raheem et al. 2018, Voloshin et al. 2016). The latter organisms have gain enormous attention recently due to their attractive advantages including high growth rate, high oil productivity, great photosynthetic efficiencies and less requirement of arable land (Bhalamurugan et al. 2018, Thawechai et al. 2016). Apart from the high oil productivity for biodiesel, its untapped potential to produce carbohydrate and protein can be explored for biogas (Ferreira et al. 2017, Tao et al. 2017) and agriculture application (Khatoon et al.
2010). Despite these advantages, the major challenges for scale-up biomass production are high capital and operational cost especially cultivation cost and high requirement for freshwater (Chia et al. 2018). Thus, more studies are needed to evaluate the strategy to remedy this bottleneck. Recently, wastewater (Acién Fernández et al. 2018, Ashokkumar et al. 2019, Wuang et al. 2016) and food waste (Chew et al. 2018, Lau et al. 2014, Zhang et al. 2018) have been formulated as cultivation medium since they are rich in nutrients which can promote microalgae growth while the microalgal growth can purify the waste before discharging into water bodies. This integration strategy renders a win-win situation by reducing environmental pollution and concurrently minimizing the cultivation cost of microalgae.

Tropical fruits are one of the important economic commodities in Malaysia. Despite consumed by Malaysian and exported to other countries, some tropical fruits are used for industrial processing for the production of fruit juice, flavouring and canned fruit. The worldwide popularity and demand for tropical fruits has shown an increasing trend from time to time (Rozhan 2017). Some tropical fruits such as pineapple and durian encompass more than 50\% rind and seeds that are not consumable. These fruits wastes are rich in moisture and organic composition therefore the long-term disposal of these fruit wastes to the environment not only results in greenhouse gas emission but also environmental pollution 
(Cheok et al. 2018). Hence, treatment of these fruit wastes before disposing of in the environment is necessary. In this context, the integration of tropical fruit waste medium for the cultivation of microalgae can be a potential strategy to solve the aforementioned problems.

The objective of this preliminary study was to investigate the potential of using tropical fruit wastes such as papaya, pineapple and mango as nutrient mediums for the cultivation of $C$. vulgaris and $H$. pluvialis. The optimum concentration for three fruit wastes was identified to evaluate the feasibility of replacing the inorganic medium with tropical fruit waste medium.

\section{MATERIALS AND METHODS}

\section{Microalgae Strain Cultivation}

Microalgae strain of $C$. vulgaris used in this study was derived from a local lake near Inti International University, while $H$. pluvialis used in this study was obtained from Algaetech Malaysia. Both microalgae were cultivated in a $250 \mathrm{~mL}$ conical flask containing $150 \mathrm{~mL}$ of Basal's basic medium (BB medium). Microalgae cultures were incubated at room temperature under illumination from cool-white fluorescent tubes for 16:8 hours of the light-dark cycle. During the cultivation, microalgae were manually shaken twice each day to prevent microalgal adherence and congregation.

Growth phases of the cells were investigated by determining cell density using a haemocytometer (MarienfeldSuperior, Neubauer) under a light microscope (Eclipse E-100 LED, Nikon).

\section{Microalgae Cultivation with Fruit Waste Medium}

Tropical organic fruits namely papaya, pineapple and mango were purchased from the local market. The fruits were washed and cut. The fruit waste including peel and core was cut, blended and filtered using a kitchen sieve. Then, the Duran bottles containing filtered fruit waste solution were heated in a microwave oven at $400 \mathrm{~W}$ for 10 minutes. The bottles were wrapped with aluminium foil and kept in the refrigerator at $4^{\circ} \mathrm{C}$ to avoid nutrient decomposition. To prepare $250 \mathrm{~mL}$ of different concentrations $(5 \%, 10 \%$, $20 \%$ and $25 \%$ ) of papaya, pineapple and mango fruit waste mediums, each fruit waste was diluted with deionized water. Each diluted fruit waste medium was immediately cultivated with $4 \mathrm{~mL}$ of 2-days-old $C$. vulgaris and 4-daysold $H$. pluvialis. Microalgae cultivated in BB medium and deionized water was used as positive and negative control respectively. The microalgae were cultivated at the condition as previously described.

\section{Determination of Microalgae Cell Growth}

The dry cell weight (DCW) of the microalgae biomass was attained by vacuum filtering $5 \mathrm{~mL}$ aliquots of culture using pre-weighted mixed cellulose ester membrane filters with absorbent pads ( $0.45 \mu \mathrm{m}$ pore size, $47 \mathrm{~mm}$ in diameter). Each loaded filter was dried at $80^{\circ} \mathrm{C}$ until the weight was constant. To obtain the dry cell weight of microalgae, the dry weight of the blank membrane filter was subtracted from that of the loaded membrane filter.

The DCW was used to calculate the microalgae growth. The biomass concentration, productivity and specific growth rate were calculated using the formulas below (Chew et al. 2018, He et al. 2018):

Biomass concentration, $\mathrm{X}=\left(\mathrm{DCW}_{\mathrm{t}} /\right.$ volume of aliquots $)$ $-\left(\mathrm{DCW}_{0} /\right.$ volume of aliquots $)$

Biomass productivity, $\mathrm{P}_{\mathrm{b}}=\left(\mathrm{X}_{\mathrm{f}}-\mathrm{X}_{0}\right) /\left(\mathrm{t}_{\mathrm{f}}-\mathrm{t}_{0}\right)$

Specific growth rate, $\mu=\left(\ln X_{f}-\ln X_{0}\right) /\left(t_{f}-t_{0}\right)$

Where $\mathrm{X}_{\mathrm{f}}$ and $\mathrm{X}_{0}$ are the biomass concentration $(\mathrm{g} / \mathrm{L}$ ) on days $t_{f}$ and $t_{0}$ (the end and beginning of the determined growth phase respectively).

\section{Statistical Analysis}

All experiments were conducted in triplicates and data were presented as means \pm standard error of the mean.

\section{RESULTS AND DISCUSSION}

Prior to microalgae cultivation with tropical fruit waste mediums, the growth phase of two microalgae in BBM was determined based on the cell count. The results showed that the $C$. vulgaris entered the log phase during the $2^{\text {nd }}$ day and then entered the stationary phase during the $4^{\text {th }}$ day while $H$. pluvialis initiated $\log$ phase during $1^{\text {st }}$ day and entered stationary phase during $8^{\text {th }}$ day. The 2 -day-old $C$. vulgaris and 4-day-old $H$. pluvialis had adequate cell density therefore they were inoculated into a $250 \mathrm{~mL}$ fruit waste medium. The inoculum of high cell density was imperative to ensure the survival of microalgae in the new environment (Gani et al. 2016).

\section{Effect of Fruit Waste Medium Concentrations on Microalgae Growth}

The growth of $C$. vulgaris and H. pluvialis in papaya, pineapple and mango fruit wastes at various dilutions and in BB medium are shown in Fig. 1 and Fig. 2. The results demonstrate that these two microalgae could grow in almost all concentrations (10,15\%,20\% and 25\%) of papaya, pineapple and mango waste mediums compared to microalgae that grew in negative control which is 
deionised water. Microalgae of $C$. vulgaris supplemented with $20 \%$ papaya, pineapple and mango yielded biomass concentration of $4.133 \mathrm{~g} / \mathrm{L}, 4.533 \mathrm{~g} / \mathrm{L}$ and $4.600 \mathrm{~g} / \mathrm{L}$ respectively at the end of 7 days which is higher than the BB medium at $2.233 \mathrm{~g} / \mathrm{L}$ (Table 1). C. vulgaris in $5 \%$ papaya, pineapple and mango waste did not grow well and the biomass concentration was very low, only at 0.667 , 1.100 and $0.533 \mathrm{~g} / \mathrm{L}$ respectively, while C. vulgaris in $25 \%$ papaya, pineapple and mango wastes yielded 1.500, 1.400 and $1.433 \mathrm{~g} / \mathrm{L}$ respectively.

As shown in Fig. 2, H. pluvialis was able to grow in all concentrations of papaya, pineapple and mango fruit waste mediums. H. pluvialis in 10\% mango waste yielded biomass concentration of $3.400 \mathrm{~g} / \mathrm{L}$ at the end of 7 days which is comparable to positive control at $3.500 \mathrm{~g} / \mathrm{L}$. Although other concentrations of fruit waste medium yielded biomass concentration that lower than BB medium, a slow and increasing trend was observed.

Papaya, pineapple and mango peels have been discovered to contain organic carbon, protein, vitamins and trace metals (Abdul Aziz et al. 2012, Souza et al. 2016, Siti Roha et al.2013, Suchiritha et al. 2017) which can be supplemented to support microalgae growth. However, the nutrient concentration must be monitored since concentrated nutrient would contrarily reduce microalgae growth. For all the concentration of fruit waste medium up to $25 \%$ including the positive control, the $C$. vulgaris grew rapidly during the first 3 days and continued to attenuate slowly until the $7^{\text {th }}$ day, indicating the fruit waste medium did not inflect the growth phase of $C$. vulgaris. The continuous decline or slow increment of biomass concentration after $3^{\text {rd }}$ day probably stemming from the diminishing of nutrients and the cells instigated the stationary phase. In Table 1 , the results showed that $C$. vulgaris in $20 \%$ fruit waste medium gave the highest biomass concentration and average biomass productivity when compared to BB medium.

For $H$. pluvialis, the cells grew slowly in BB medium and fruit waste mediums within $7^{\text {th }}$ day. As shown in Table 2, H. pluvialis in 5\% and $10 \%$ fruit waste mediums resulted in higher biomass concentration and productivity, indicating that lower fruit waste mediums are more suitable for $H$. pluvialis to grow. Compared to $H$. pluvialis, C. vulgaris showed higher biomass concentration and biomass productivity. It is probably because of their varied metabolisms mode. Besides, compared to C. vulgaris, $H$. pluvialis preferred growing in a low concentration of fruit waste medium. The high turbidity of the fruit waste medium may force microalgae to grow in heterotrophic or mixotrophic mode. Testaments from previous studies have revealed $C$. vulgaris was capable to grow with higher biomass concentration in various types of mixotrophic or heterotrophic medium (Gao et al. 2019, Lam et al. 2017, Li et al. 2019, Melo et al. 2018), whereas not many studies have unveiled capability of $\mathrm{H}$. pluvialis in different types of mixotrophic medium (Sipaúba-Tavares et al. 2015). Further
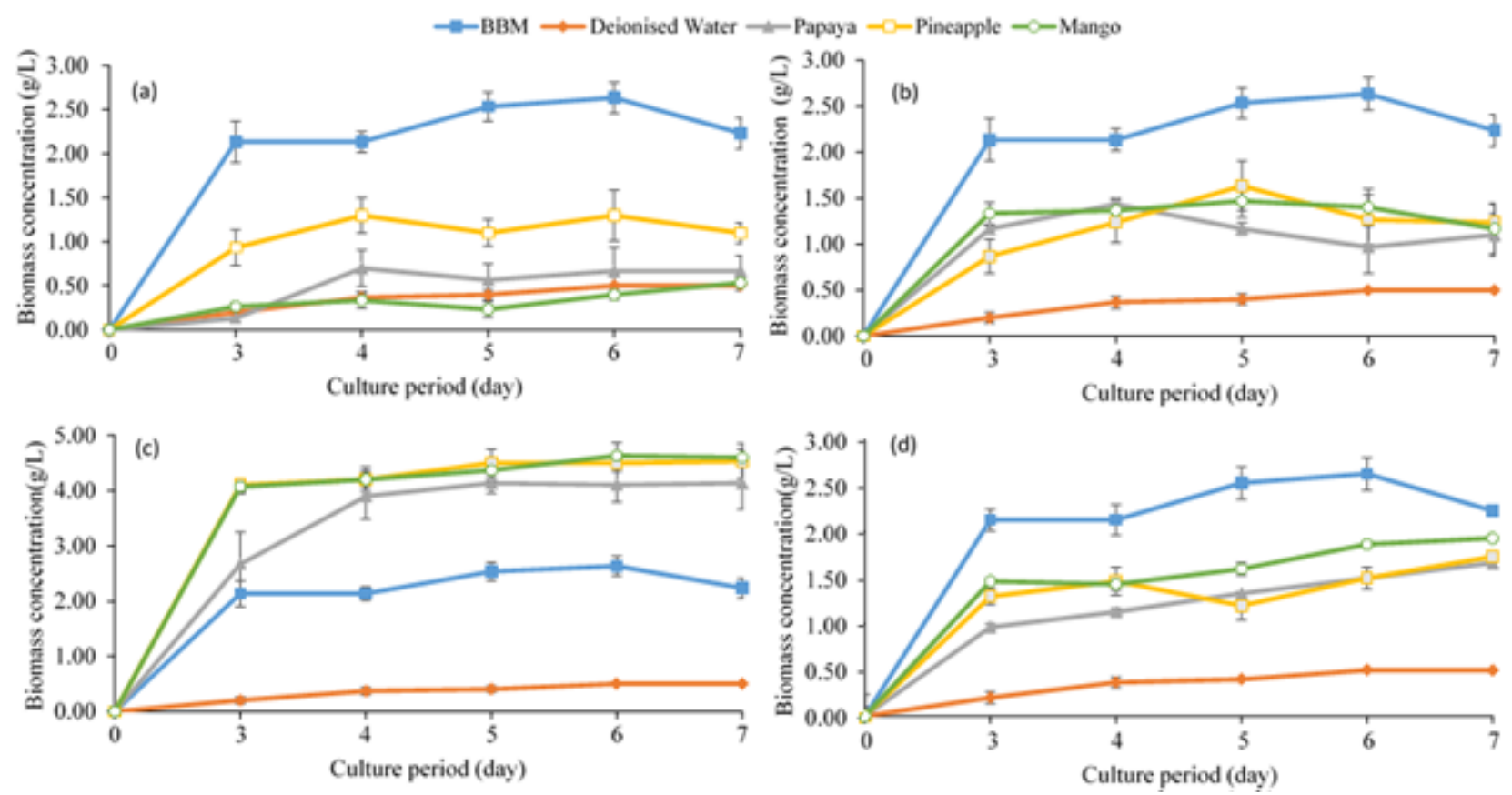

Fig. 1: Biomass concentration of C. vulgaris in (a) $5 \%$, (b) $10 \%$, (c) $20 \%$ and (d) $25 \%$ papaya, pineapple and mango wastes and in BB medium (n $=3$ ). 

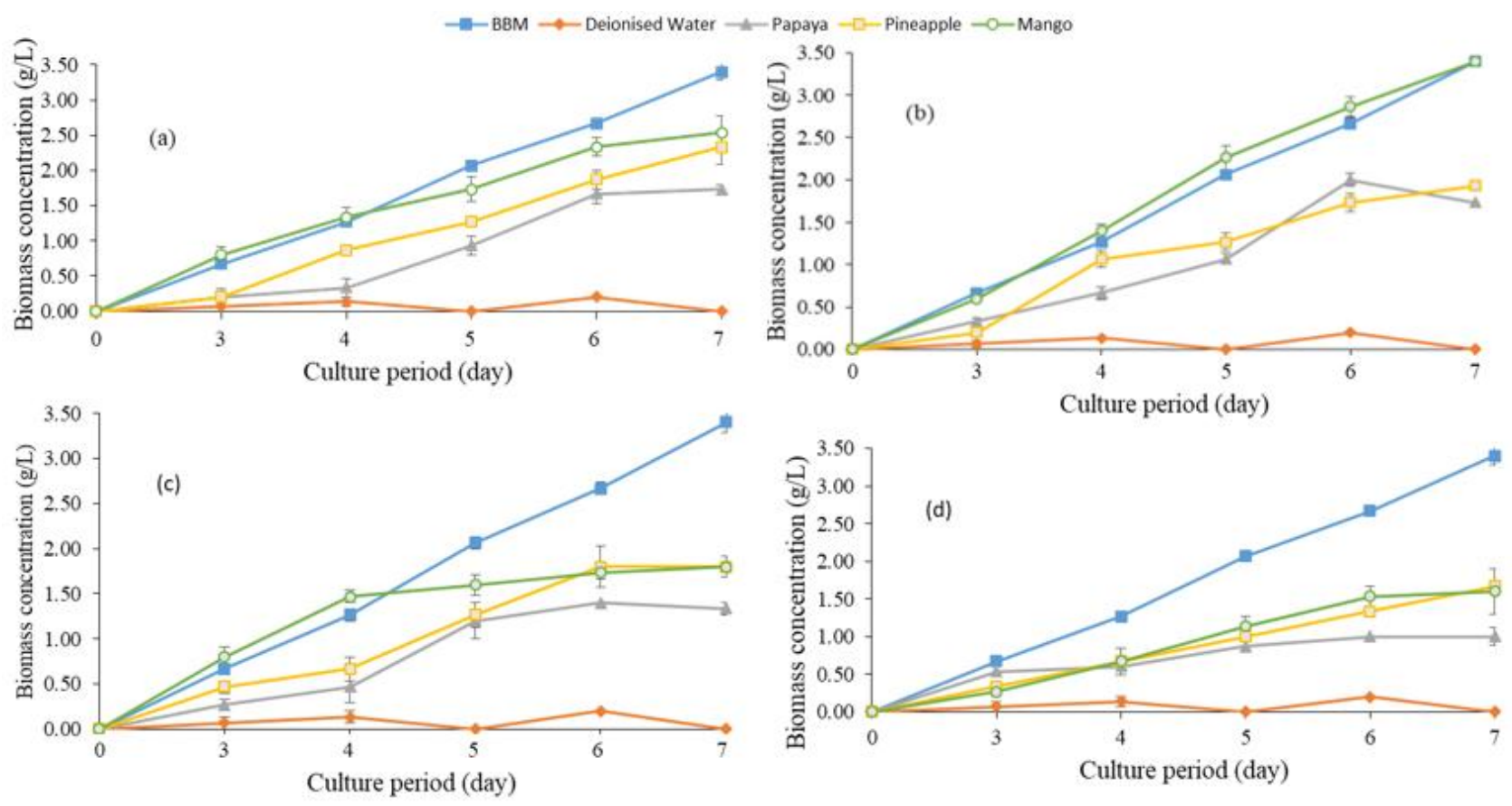

Fig. 2: Biomass concentration of H. pluviaris in (a) $5 \%$, (b) $10 \%$, (c) $20 \%$ and (d) $25 \%$ papaya, pineapple and mango wastes and in BB medium $(n=3)$.

Table 1: Final dry cell weight (DCW), average biomass productivity $\left(\mathrm{P}_{\mathrm{b}}\right)$ and average specific growth rates $(\mu)$ of $C$. vulgaris in papaya, pineapple and mango waste medium at various concentrations and in BB medium.

\begin{tabular}{|c|c|c|c|c|}
\hline Medium & & DCW $(\mathrm{g} / \mathrm{L})$ & $\mathrm{P}_{\mathrm{b}}$ & $\mu\left(d^{-1}\right)$ \\
\hline BBM & & $2.233 \pm 0.176$ & $0.172 \pm 0.015$ & $0.180 \pm 0.022$ \\
\hline DW & & $0.500 \pm 0.000$ & $0.022 \pm 0.008$ & $0.046 \pm 0.003$ \\
\hline \multirow[t]{4}{*}{ Papaya } & $5 \%$ & $0.667 \pm 0.176$ & $0.045 \pm 0.013$ & $0.040 \pm 0.007$ \\
\hline & $10 \%$ & $1.100 \pm 0.231$ & $0.110 \pm 0.013$ & $0.067 \pm 0.012$ \\
\hline & $20 \%$ & $4.133 \pm 0.470$ & $0.244 \pm 0.047$ & $0.208 \pm 0.021$ \\
\hline & $25 \%$ & $1.667 \pm 0.333$ & $0.076 \pm 0.006$ & $0.145 \pm 0.016$ \\
\hline \multirow[t]{4}{*}{ Pineapple } & $5 \%$ & $1.100 \pm 0.115$ & $0.084 \pm 0.015$ & $0.105 \pm 0.013$ \\
\hline & $10 \%$ & $1.233 \pm 0.203$ & $0.089 \pm 0.009$ & $0.069 \pm 0.008$ \\
\hline & $20 \%$ & $4.533 \pm 0.318$ & $0.319 \pm 0.009$ & $0.240 \pm 0.034$ \\
\hline & $25 \%$ & $1.733 \pm 0.120$ & $0.096 \pm 0.005$ & $0.164 \pm 0.023$ \\
\hline \multirow[t]{4}{*}{ Mango } & $5 \%$ & $0.533 \pm 0.088$ & $0.025 \pm 0.004$ & $0.046 \pm 0.007$ \\
\hline & $10 \%$ & $1.167 \pm 0.273$ & $0.113 \pm 0.003$ & $0.117 \pm 0.004$ \\
\hline & $20 \%$ & $4.600 \pm 0.153$ & $0.308 \pm 0.005$ & $0.365 \pm 0.006$ \\
\hline & $25 \%$ & $1.933 \pm 0.030$ & $0.103 \pm 0.007$ & $0.101 \pm 0.012$ \\
\hline
\end{tabular}

research is required to discover the influence of metabolism mode on the growth of $H$. pluvialis. Moreover, $C$. vulgaris has a shorter $\log$ phase than $H$. pluvialis, indicating $C$. vulgaris has faster cell metabolism thereby their cell concentration grow quickly. Greater cultivation time is feasibly required for $H$. pluvialis to achieve higher biomass concentration and average specific growth rate.
The average specific growth rates ( $\mu$ ) during 3-7 days' culture are shown in Table 1 . The increasing of papaya and mango waste medium concentration stimulated $\mu$ of $C$. vulgaris to increase until the concentration of $20 \%$. $\mu$ was dropped in the concentration of $10 \%$ pineapple and then increased in the concentration of $20 \%$ followed by a decrease in the concentration of $25 \%$. $\mu$ attained in papaya, pineapple 
Table 2: Final dry cell weight (DCW), average biomass productivity $\left(\mathrm{P}_{\mathrm{b}}\right)$ and average specific growth rates $(\mu)$ of $H$. pluviaris in papaya, pineapple and mango waste medium at various concentrations and in BB medium.

\begin{tabular}{|c|c|c|c|c|}
\hline Medium & & DCW $(g / L)$ & $\mathrm{P}_{\mathrm{b}}$ & $\mu\left(d^{-1}\right)$ \\
\hline BBM & & $3.400 \pm 0.115$ & $0.314 \pm 0.013$ & $0.239 \pm 0.009$ \\
\hline DW & & $0.000 \pm 0.000$ & $0.015 \pm 0.006$ & $0.022 \pm 0.007$ \\
\hline \multirow[t]{4}{*}{ Papaya } & $5 \%$ & $1.733 \pm 0.067$ & $0.144 \pm 0.022$ & $0.102 \pm 0.018$ \\
\hline & $10 \%$ & $1.733 \pm 0.133$ & $0.179 \pm 0.017$ & $0.133 \pm 0.014$ \\
\hline & $20 \%$ & $1.333 \pm 0.115$ & $0.145 \pm 0.033$ & $0.090 \pm 0.001$ \\
\hline & $25 \%$ & $1.000 \pm 0.200$ & $0.135 \pm 0.023$ & $0.046 \pm 0.002$ \\
\hline \multirow[t]{4}{*}{ Pineapple } & $5 \%$ & $2.333 \pm 0.240$ & $0.197 \pm 0.021$ & $0.125 \pm 0.019$ \\
\hline & $10 \%$ & $1.933 \pm 0.176$ & $0.192 \pm 0.022$ & $0.111 \pm 0.017$ \\
\hline & $20 \%$ & $1.800 \pm 0.200$ & $0.189 \pm 0.040$ & $0.077 \pm 0.005$ \\
\hline & $25 \%$ & $1.667 \pm 0.115$ & $0.156 \pm 0.017$ & $0.062 \pm 0.006$ \\
\hline \multirow[t]{4}{*}{ Mango } & $5 \%$ & $2.533 \pm 0.240$ & $0.283 \pm 0.027$ & $0.157 \pm 0.004$ \\
\hline & $10 \%$ & $3.400 \pm 0.200$ & $0.328 \pm 0.022$ & $0.161 \pm 0.014$ \\
\hline & $20 \%$ & $1.733 \pm 0.115$ & $0.250 \pm 0.026$ & $0.030 \pm 0.001$ \\
\hline & $25 \%$ & $1.600 \pm 0.429$ & $0.161 \pm 0.045$ & $0.033 \pm 0.010$ \\
\hline
\end{tabular}

and mango waste mediums with the concentration of $20 \%$ were higher than in BB medium.

For H. pluvialis, $\mu$ was increased from the concentration of $5 \%$ to $10 \%$ and then decreased in the concentration of $20 \%$ and $25 \%$. These results indicated that fruit waste with a concentration of $10 \%$ was ideal for $H$. pluvialis to grow compared with other concentrations while it was not optimised as in BB medium.

This research confirmed that some of the diluted papaya, pineapple and mango waste mediums were capable to grow these two microalgae. Similar results were reported by previous studies (Chew et al. 2018, Heller et al. 2015, Lau et al. 2014, Zhang et al. 2018), using food waste as a nutrient medium for $C$. vulgaris. A high concentration of food waste exhibited an inhibition effect on cell growth while a low concentration of food compost favoured the cell growth. In this study, only $20 \%$ of fruit waste medium with C. vulgaris have higher biomass concentration, productivity and specific growth rate than those in BB medium. Whereas only $H$. pluvialis in $10 \%$ mango waste medium has similar biomass concentration, productivity and specific growth rate compared to those in $\mathrm{BB}$ medium. Two reasons can elucidate this phenomenon. First, a high level of turbidity was observed in all fruit waste mediums, and the sediment may be a light barrier for microalgae cultivation. The high turbidity and sediment hinder the light to penetrate the fruit waste medium therefore microalgae were distracted from photosynthesis. This can be confirmed by comparing pigments colour of microalgae that grew in BB medium and fruit waste medium. The cell pigment in the fruit waste medium was paler than those grown in the BB medium. Furthermore, the fruit product usually has a somewhat acidic $\mathrm{pH}$ which may not favour most microalgae growth (Difusa et al. 2015, Zhang et al. 2014).

Second, to reduce the cultivation cost, raw and unsterile fruit waste was used in the present study. As it was unsterile, unknown zooplanktons appeared. The presence of zooplanktons competed for nutrient sources thereby reduced the number and biomass concentration of algal cells. Besides, white fungi appeared in all concentration of papaya waste mediums during cultivation. Although combating white fungi was attempted to be removed using spatula, it regrew again and only white fungi in $20 \%$ papaya waste medium that growing $C$. vulgaris was successfully eliminated.

\section{CONCLUSIONS}

In this study, the recycling of nutrients from tropical fruit waste medium was investigated by the cultivation of $C$. vulgaris and $H$. pluvialis using papaya, pineapple and mango wastes with different concentrations. The highest biomass concentration for C. vulgaris and H. pluvialis was attained in $20 \%$ fruit waste mediums and $10 \%$ mango fruit waste respectively. This study demonstrated that papaya, pineapple and mango wastes have feasible to cultivate microalgae. The utilization of tropical fruit waste medium can be an effective strategy to minimize environmental pollution and the microalgae cultivation cost. Determination of nutrient removal rate and metabolites such as lipids, carbohydrates and proteins should be carried out in the future to investigate the economic value of this integrated strategy. 


\section{ACKNOWLEDGEMENT}

The authors would like to acknowledge UNITEN BOLD2025 Phase 2 Grant No. "10436494/B/2019006" for the financial support.

\section{REFERENCES}

Abdul Aziz, N.A., Wong, L.M., Bhat, R. and Cheng, L.H. 2012. Evaluation of processed green and ripe mango peel and pulp flours (Mangifera indica var. Chokanan) in terms of chemical composition, antioxidant compounds and functional properties. J. Sci. Food Agric., 92(3): 557-563.

Acién Fernández, F.G., Gómez-Serrano, C. and Fernández-Sevilla, J.M. 2018. Recovery of nutrients from wastewaters using microalgae. Front. Sustain. Food Syst., 2: 1-13.

Ashokkumar, V. Chen, W.H., Kamyab, H., Kumar, G., Al-Muhtaseb, A.H. and Ngamcharussrivichai, C. 2019. Cultivation of microalgae Chlorella sp. In municipal sewage for biofuel production and utilization of biochar derived from residue for the conversion of hematite iron ore $\left(\mathrm{Fe}_{2} \mathrm{O}_{3}\right)$ to iron $(\mathrm{Fe})$ - Integrated algal biorefinery. Energy, 189: 116128.

Bhalamurugan, G.L., Valerie, O. and Mark, L. 2018. Valuable bioproducts obtained from microalgal biomass and their commercial applications: A review. Environmental Engineering Research, 23(3): 229-241.

BP. 2019. BP Statistical Review of World Energy 2019. In BP. London.

Chia, S.R., Chew, K.W., Show, P.L., Yap, Y.J., Ong, H.C., Ling, T.C. and Chang, J.S. 2018. Analysis of economic and environmental aspects of microalgae biorefinery for biofuels production: A Review. Biotechnol. J., 13(6): 170068.

Cheok, C.Y., Mohd Adzahan, N., Abdul Rahman, R., Zainal Abedin, N.H., Hussain, N., Sulaiman, R. and Chong, G.H. 2018. Current trends of tropical fruit waste utilization. Crit. Rev. Food Sci. Nutr., 58(3): 335-361.

Chew, K.W., Chia, S.R., Show, P.L., Ling, T.C., Arya, S.S. and Chang, J.S. 2018. Food waste compost as an organic nutrient source for the cultivation of Chlorella vulgaris. Bioresour. Technol., 267: 356-362.

Difusa, A., Talukdar, J., Kalita, M.C., Mohanty, K. and Goud, V.V. 2015. Effect of light intensity and $\mathrm{pH}$ condition on the growth, biomass and lipid content of microalgae Scenedesmus species. Biofuels, 6: 37-44.

Ferreira, A., Ribeiro, B., Marques, P.A.S.S., Ferreira, A.F., Dias, A.P., Pinheiro, H.M. et al. 2017. Scenedesmus obliquus mediated brewery wastewater remediation and $\mathrm{CO}_{2}$ biofixation for green energy purposes. J. Clean. Prod., 165: 1316-1327.

Gani, P., Sunar, N.M., Latiff, A.A.A. and Razak, A. 2016. Influence of initial cell concentrations on the growth rate and biomass productivity of microalgae in domestic wastewater. Appl. Ecol. Environ. Res., 14(2): 399-409.

Gao, F., Yang, H.L., Li, C., Peng, Y.Y., Lu, M.M., Jin, W.H., Bao, J.J. and Guo, Y.M. 2019. Effect of organic carbon to nitrogen ratio in wastewater on growth, nutrient uptake and lipid accumulation of a mixotrophic microalgae Chlorella sp. Bioresour. Technol., 282: 118-124.

He, Q.N., Yang., H.J. and Hu, C.X. 2018. Effects of temperature and its combination with high light intensity on lipid production of Monoraphidium dybowskii Y2 from semi-arid desert areas. Bioresour. Technol., 265: 407- 414.

Heller, W.P., Kissinger, K.R., Matsumoto, T.K. and Keith, L.M. 2015. Utilization of papaya waste and oil production by Chlorella protothecoides. Algal Res., 12: 156-160.

International Energy Agency, 2019. World Energy Outlook 2019. International Energy Agency.
Khatoon, N., Sengupta, P., Homechaudhuri, S. and Pal, R. 2010. Evaluation of algae based feed in goldfish (Carassius auratus) nutrition. Proc. Zool. Soc., 63(2): 109-114.

Lam, M.K., Yusoff, M.I., Uemura, Y., Lim, J.W., Khoo, C.G., Lee, K.T. and Ong, H.C. 2017. Cultivation of Chlorella vulgaris using nutrients source from domestic wastewater for biodiesel production: Growth condition and kinetic studies. Renew. Energy, 103: 197-207.

Lau, K.Y., Pleissner, D. and Lin, C.S.K. 2014. Recycling of food waste as nutrients in Chlorella vulgaris cultivation. Bioresour. Technol., 170: $144-151$.

Leong, W.H., Lim, J.W., Lam, M.K., Uemura, Y. and Ho, Y.C. 2018. Third generation biofuels: A nutritional perspective in enhancing microbial lipid production. Renew. Sust. Energ. Rev., 91: 950-961.

Li, H., Zhang, Y., Liu, J., Shen, Z., Li, A., Ma, T., Feng, Q. and Sun, Y. 2019. Treatment of high-nitrate wastewater mixtures from $\mathrm{MnO}_{2}$ industry by Chlorella vulgaris. Bioresour. Technol., 291: 121836.

Melo, R.G., Andrade, A.F., Bezerra, R.P., Correia, D.S., Souza, V.C., Brasileiro-Vidal, A.C., Marques, D.A.V. and Porto, A.L.F. 2018. Chlorella vulgaris mixotrophic growth enhanced biomass productivity and reduced toxicity from agro-industrial by-products. Chemosphere, 204: 344-350.

Raheem, A., Prinsen, P., Vuppaladadiyam, A.K., Zhao, M. and Luque, R. 2018. A review on sustainable microalgae based biofuel and bioenergy production: Recent developments. J. Clean. Prod., 181: 42-59.

Rozhan, A.D. 2017. Trends in Production, Trade, and Consumption of Tropical Fruit in Malaysia. FFTC Agricultural Policy Platform, 1-8.

Sipaúba-Tavares, L.H., Berchielli-Morais, F.A. and Scardoeli-Truzzi, B. 2015. Growth of Haematococcus pluvialis Flotow in alternative media. Braz. J. Biol., 75(4): 796-803.

Siti Roha, A.M., Zainal, S., Noriham, A. and Nadzirah, K. 2013. Determination of sugar content in pineapple waste variety N36. Int. Food Res. J., 20(4): 1941-1943.

Souza, R.A.T., Fonseca, T.A.B., Kirsch, L.S., Silva, L.S.C., Alecrim, M.M., Filho, R.F.C. and Teixeira, M.F.S. 2016. Nutritional composition of bioproducts generated from semi-solid fermentation of pineapple peel by edible mushrooms. Afr. J. Biotechnol., 15(12): 451-457.

Suchiritha, D.S., Suneetha, J.W. and Durga Rani, C.V. 2017. Nutritional properties of papaya peel. Pharma. Innov., 170(67): 170-173.

Tao, R., Kinnunen, V., Praveenkumar, R., Lakaniemi, A.M. and Rintala, J. A. 2017. Comparison of Scenedesmus acuminatus and Chlorella vulgaris cultivation in liquid digestates from anaerobic digestion of pulp and paper industry and municipal wastewater treatment sludge. J. Appl. Phycol., 29(6): 2845-2856.

Thawechai, T., Cheirsilp, B., Louhasakul, Y., Boonsawang, P. and Prasertsan, P. 2016. Mitigation of carbon dioxide by oleaginous microalgae for lipids and pigments production: Effect of light illumination and carbon dioxide feeding strategies. Bioresour. Technol., 219: 139-149.

Voloshin, R.A., Rodionova, M.V., Zharmukhamedov, S.K., Nejat Veziroglu, T. and Allakhverdiev, S.I. 2016. Review: Biofuel production from plant and algal biomass. Int. J. Hydrogen Energy, 41(39): 17257-17273.

Wuang, S.C., Khin, M.C., Chua, P.Q.D. and Luo, Y.D. 2016. Use of Spirulina biomass produced from treatment of aquaculture wastewater as agricultural fertilizers. Algal Res., 15: 59-64.

Zhang, L., Cheng, J., Pei, H., Pan, J., Jiang, L., Hou, Q. and Han, F. 2018. Cultivation of microalgae using anaerobically digested effluent from kitchen waste as a nutrient source for biodiesel production. Renew. Energy, 115: 276-287.

Zhang, Q., Wang, T. and Hong, Y. 2014. Investigation of initial pH effects on growth of an oleaginous microalgae Chlorella sp. HQ for lipid production and nutrient uptake. Water Sci. Technol., 70(4): 712-719. 